\title{
A presença de alcalóides em espécies botânicas da Amazônia. VII - Apocynaceae(1)
}

\author{
A. I. da Rocha $\left.{ }^{2}\right)$ \\ A. I. Reis Luz ( $\left.{ }^{3}\right)$ \\ W. A. Rodrigues $\left({ }^{3}\right)$
}

\begin{abstract}
Resumo
Este trabalho apresenta uma lista de espécies da família Apocynaceae, que possuem representantes no herbário do Instituto Nacional de Pesquisas da Amazônia, com os respectivos resultados do teste para indicação da presença ou não de alcalóides. As características químico-alcaloídicas dessa familia são também discutidas, com ênfase, nos gêneros para a América.
\end{abstract}

\section{INTRODUÇÃO}

Em trabalhos anteriores, apresentamos um "screening" de alcalóides em espécies botânicas da Amazônia, registradas no herbário do INPA-Manaus (Rocha et al., 1968) e uma focalização da família Annonaceae (Rocha et al., 1981). Agora, focalizamos a familia Apocynaceae, objetivando, como no caso anterior, estabelecer o conhecimento químico-alcaloídico do táxon em geral e, em particular, de seus representantes na Amazônia.

\section{APOCYNACEAR}

A família Apocynaceae é constituída por, aproximadamente, 250 gêneros e 2.000 espécies. Filogeneticamente, pertence à ordem Gentianales, subclasse Asteridae, constituindo-se num dos grupos mais evoluídos de Dicotiledôneas, com um relacionamento próximo com famílias como Asclepiadaceae, Gentianaceae e Loganiaceae. Fitogeograficamente, suas espécies se distribuem predominantemente pelas regiões tropicais e subtropicais de todo mundo e, ocasionalmente, nas regiões temperadas. No continente americano, são ci- tados 68 gêneros, dos quais 32 na Amazônia (Quadro I). E destes, 30 estão representados no herbário do INPA-Manaus (Quadro II). Do ponto de vista químico-alcaloídico, temos informações de aproximadamente 50 gêneros, dos quais apenas 11 existem no herbário do INPA (Quadro II).

Os alcalóides indólicos de plantas contam hoje aproximadamente 1.200 e com respeito aos seus aspectos estruturais são divididos em duas classes: uma, mais simpies, que apresenta o núcleo indólico ou um de seus derivados como característica comum e são distribuídos em diversas famílias: Apocynaceae, Bignoniaceae, Calycanthaceae, Combretaceae, Cyperaceae, Eleagnaceae, Gramineae, Lauraceae, Loganiaceae, Leguminosae, Malpighiaceae, Myristicaceae, Ochnaceae, Palmae, Papaveraceae, Passifloraceae, Polyganaceae, Rubiaceae, Rutaceae, Sapotaceae, Simaroubaceae, Solanaceae, Symplocaceae, Tiliaceae e Zygophyllaceae (Allen \& Holmstedt, 1980), pelo que parecem não ter significado taxonômico; e outra que apresenta, além do núcleo triptamínico, um núcleo monoterpênico da secologanina, tem ocorrência restrita às famílias Apocynaceae, Loganiaceae e Rubiaceae e é adequável para estudos quimiossistemáticos (Rocha, 1977).

Nas Apocynaceas, os alcalóides indoloterpênicos têm sido isolados de uma única subfamília, a Plumerioideae, que é formada por quatro tribos e 32 gêneros. Chama a atenção o fato de que muitas espécies da família Apocynaceae são pouco conhecidas e algumas são confundidas com outras. Vale mencionar

(1) - Pesquisa financiada pelo Banco da Amazônia S.A.

(2) - Universidade do Amazonas, Manaus.

(3) - Instituto Nacional de Pesquisas da Amazônia, Manaus. 
que a tribo Tabernaemontaneae tem sido objeto de discussão por mais de um século (Markgraf, 1976; Pichon, 1948), e o número de nomes genéricos que aparecem na literatura química como sinônimos de Tabernaemontana (Pagiantha, Rejoua, Ervatamia, Hazunta, Peschiera, Conopharyngia, Pandaca, Gabunia) é Impressionante.

Ressalte-se, também, que alguns especialistas (Melchior, 1964), consideram esta tribo como uma subfamília, Tabernaemontanoideae. Do ponto de vista químico, a situação também

\section{QUADRO I - Gêneros de Apocynaceae que ocorrem na América}

\begin{tabular}{ll} 
Allamanda (12) - A & Malouetia (30) - A \\
Allomarkgrafia (4) & Mandevilla (130) - A \\
Ambelania (3) (incl. Neocou- & Mesechites (6) - A \\
ma) (11) - A & Microplumeria (1) - A \\
Amsonia (7) & Molongum (6) \\
Anacampta (= Tabernaemon- & Mortoniella (1) \\
tana) (12) - A & Neurolobium (1) \\
Anartia (= Tabernaemontana) & Odontadenia (26) - A \\
(4) - A & Parahancornia (2) - A \\
Anechites (1) & Paralyxia (2) \\
Angadenia (2) & Peltastes (8) \\
Apocynum (7) & Peschiera (= Tabernae- \\
Asketanthera (4) & montana) (18) - A \\
Aspidosperma (80) - A & Plumeria (7) \\
Bonafousia (=Tabernaemon- & Plumeriopsis (1) \\
tana) (10) - A & Prestonia (68) - A \\
Ceratites (1) & Rauvolfia (90) - A \\
Condylocarpon (12) & Rhabdadenia (3) - A \\
Couma (6) - A & Rhipidia (1) - A \\
Cycladenia (1) & Rhodocalyx (1) \\
Echites (6) & Salpinctes (2) - A \\
Elytropus (1) & Secondatia (6) - A \\
Fernaldia (2) & Skytanthus (2) \\
Forsteronia (50) - A & Stemmadenia (23) - A \\
Galactophora (5) - A & Stenosolen (7) - A \\
Geissospermum (3) - A & Stipecoma (1) \\
Hancornia (1) - A & Strempeliopsis (1) \\
Haplophyton (1) & Taberna (1) \\
Himatanthus (7) - A & Tabernaemontana (80) \\
Lacmellea (18) - A & Temnadenia (4) \\
Landolphia (3) - A & Thevetia (8) \\
Laubertia (4) & Thenardia (4) \\
Laxoplumeria (1) - A & Trachelospermum (24) \\
Lochnera (3) & Urechites (2) \\
Macropharynx (12) & Woytkowskia (1) - A \\
Macoubea (6) - A & Vallesia (8) \\
Macrosiphonią (10) & \\
& \\
\hline &
\end{tabular}

A - Gêneros que ocorrem na Amazônia.

() - Número de espécies.
QUADRO II - Gêneros de Apocynaceae que ocorrem na Amazônia

Estudados p/ alcalóides

Anacampta $(=$ Tabernaemontana $)$

Aspidosperma

Geissospermum

Microplumeria

Malouetia

Peschiera (= Tabernaemontana)

Prestonia

Rauwolfia

Rhabdadenia

Stenosolen

Stemmadenia

Não estudados p/ alcalóides

Ambelania

Allamanda (-)

Anartia ( = Tabernaemontana) $\left({ }^{*}\right)$

Bonafousia (= Tabernaemontana) $\left({ }^{*}\right)$

Couma (-)

Forsteronia (-)

Galactophora (-)

Hancornia (+)

Himatanthus $(+)$

Lacmella $(+)$

Landolphia ("*)

Laxoplumeria (")

Mandevilla $(+)$

Macoubea (+)

Mesechites (")

Odontadenia $(+)$

Parahancornia $(+)$

Rhipidia (+)

Secondatia $\left({ }^{*}\right)$

Salpinctes (")

Woytkowskia (**)

$(+)$ - Gêneros com testes positivos para alcalóides.

(-) - Gêneros com testes negativos para alcalóides.

(*) - Gêneros näo testados para alcalóides.

(*) - Näo têm registro no INPA.

é crucial porque embora os seus alcalóides tenham a mesma origem biogenética dos das demais tribos de Plumerioideae, neste taxon os alcalóides do tipo iboga são marcantes, e só não exclusivos face aos registros feitos nos gêneros Alstonia (Croqueiois et al., 1972) e Catharanthus (Hesse, 1964 e 1968).

Os alcalóides esteroidais em Apocynaceae caracterizam a subfamília Apocynoideae (Echitoideae). Um aspecto comum destes compostos é um anel heterocíclico de cinco membros 
adjacentes ao anel D do esqueleto esteroidal, envolvendo os átomos, C-17, C-13, C-18. Este sistema heterocíclico pode ser tanto lactônico com a carbonila em C-18 como pirrolidínico, ou $\triangle^{\prime}$-pirrolina. Em C-13, do esqueleto esteroidal, tem-se tanto funções oxigenadas como aminadas (Habermehl, 1973).

$\mathrm{Na}$ subfamília Cerberoideae, têm sido determinados alcalóides piridínicos e piperidínicos (Raffauf, 1970). Observe-se que no gênero Strophantus, classificado por Dalla Torre \&
Harms (1963) na subfamília Echitoideae, tribo Echitideae, e por Melchior (1964) na subfamília Echitoideae, tribo Nerieae, também temos indicada a presença de alcalóides piridínicas (Raffauf, 1970), pelo que sugerimos o seu estudo com vistas ao seu relacionamento com os demais gêneros de Cerberoideae.

As espécies de Apocynaceae registradas no herbário do INPA-Manaus e os resultados dos testes para alcalóides constam do Quadro III.

\section{QUADRO III - Apocynaceae}

N. HERBARIO

NOME CIENTÍFICO

FOLHA

CAULE

\section{Allamanda}

80351

catharthica L.

nobilis $T$. Moore

\section{Ambelania}

7662

3727

9536

16782

12944

8438

5618

9699

8505

6903

9632

14238

15064
8160
6
15546
10575

A presença... acida Aubl. cuneata Müll. Arg. duckei Mgf. grandiflora Huber laxa Müll. Arg. longiloba Mgf. markgrafiana Monach. quadrangularis Müll. Arg. tenuiflora Müll. Arg.

\section{Anacampta}

angulata (Mart. ex Müll. Arg.) Miers coriacea (Link.) Mgf.

kuhlmannii Mgf. macrocalyx (Müll. Arg.) Mgf.

maxima Mgf.

pendula Mgf.

rigida (Miers) Mgf.

rupicola (Benth.) Mgf.

rupicola (Benth.) Mgf. var. oblongifolia Müll. Arg.

rupicola (Benth.) Mgf. var. sprucei Müll. Arg.

submollis (Mart.) Miers.

riedelii (M. Arg.) Mgf.

$$
++
$$$$
+++
$$
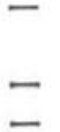

$+$

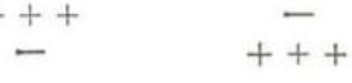

Anartia

attenuata (Miers) Mgf.

flavicans (R.E. Sch.) Miers

\section{Aspidosperma}

album (Vahl) R. Ben.

carapanaúba Pichon

$\stackrel{+}{+}+$

desmanthum Benth.

eteanum Mgf.

exalatum Monach. 
macrocarpon Mart.

multiflorum DC.

8125

17575

14746

7081

15075

15141

13160

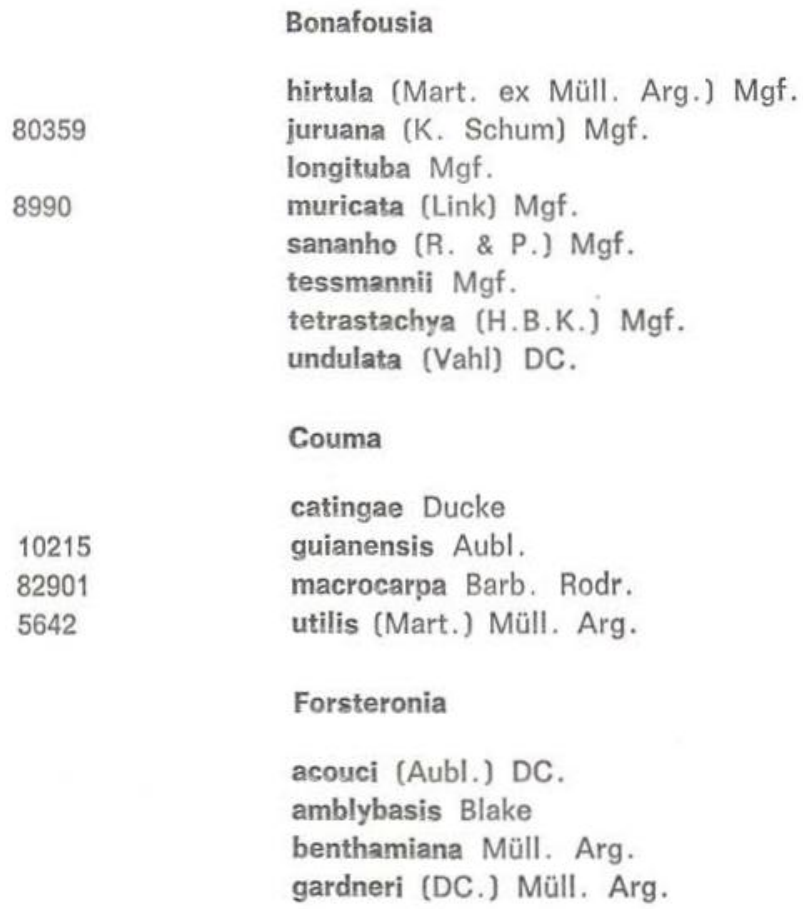

juruana (K. Schum) Mgf.

longituba Mgf.

muricata (Link) Mgf.

sananho (R. \& P.) Mgf.

tessmannii Mgf.

tetrastachya (H.B.K.) Mgf,

undulata (Vahl) DC.

Couma

catingae Ducke

guianensis Aubl.

macrocarpa Barb. Rodr.

utilis (Mart.) Müll. Arg.

\section{Forsteronia}

acouci (Aubl.) DC.

amblybasis Blake

benthamiana Müll. Arg.

gardneri (DC.) Müll. Arg.

oblongum DC.

obscurinervium Azamb.

rigidum Rusby (laxiflorum Kuhlm.)

spruceana Benth.

subincanum Mart.

ulei Mgf.

verruculosum Müll. Arg.

williansii A.P. Duarte

paniculatum Azamb.

aquaticum Ducke

\section{Forsteronia}

gracilis (Benth.) Müll. Arg.

laurifolia (Benth.) DC.

mollis Rusby

\section{Galactophora}

Calycina (Huber) Woods.

crassifolia Woods.

\section{Geissospermum}

argentum Woods.

sericeum Benth. \& Hook.

\section{Hancornia}

amapa Huber laeve (Vell.) Baill. (G. Vellozii Fr. All.)

$$
\begin{aligned}
& +++ \\
& +++ \\
& +++
\end{aligned}
$$


Himatanthus

articulatus (Vahl) Woods.

attenuatus (Müll. Arg.) Woods.

bracteatus (DC) Woods.

lancifolius (Müll. Arg.) Woods.

(Plumeria lancifolia Mart.)

phagedaenicus (Mart.) Woods.

sucuuba (Spruce) Woods.

Lacmellea

aculeata (Ducke) Monach.

arborescens (Müll. Arg.) Mgf. var. arborescens

arborescens (Müll. Arg.) Mgf. var. monosperma

(Müll. Arg.) Mgf.

floribunda (Poepp) Benth.

gracilis (Müll. Arg.) Monach.

latescens (Kuhlm.) Mgf.

ramosissima (Müll. Arg. in Mart.) Mgf.

\section{Laxoplumeria}

macrophylla (Kuhlm.) Monach.

\section{Macoubea}

guianensis Aubl.

sprucei (Müll. Arg.) Mgf.

\section{Malouetia}

amplexicaulis Müll. Arg.

duckei Mgf.

furfuracea Spruce

lata Mgf.

mexiae Woods.

tamaquarina (Aubl.) DC.

virescens Spruce

\section{Mandevilla}

hirsuta (A. Rich.) K. Schum.

krukovii Woods.

lasiocarpa (DC.) Malme

leptophyila (DC.) K. Schum.

rugosa (Benth.) Woods.

scaberula N.E. Brown

scabra (Roem. \& Schult.) K. Schum.

schlimii (M. Arg.) Woods.

subcarnosa (Bth.) Woods.

symphytocarpa (G. W. Mayer) Woods.

ulei K. Schum.

\section{Microplumeria}

anomala (M. Arg.) Mgf.

\section{Mesechites}

bicornulata (Rusby) Woods.

trifida (Jacq.) Müll. Arg. 
Odontadenia

9987

cognata (Stadelm.) Woods.

funigera Woods.

geminata (Roem. \& Scult.) Müll. Arg.

glauca Woods.

hoffmannseggiana (Steud.) Woods.

hypoglauca (Stadelm.) Müll. Arg.

laxiflora (Rusby) Woods.

neglecta Woods.

nitida (Vahl.) Müll. Arg.

perrottetii (DC.) Woods.

puncticulosa (A. Rich.) Pulle

spoliata Malme

stemmadeniifolia Woods.

verrucosa (Roem. \& Schult.) K. Schum.

Parahancornia

amapa (Huber) Ducke

amara Monach.

krukovii Monach.

negrensis Monach.

peruviana Monach.

tabernaemontana Woods.

sprucei (M. Arg.) Mgf.

Plumeria

cuneata $\mathrm{Sm}$.

Peschiera

arcuata (R. \& P.) Mgf.

myriantha (Britt.) Mgf.

psychotriifolia (H.B.K.) Miers

stenantha Mgf.

\section{Prestonia}

acutifolia (Benth.) K. Schum.

coalita (Vell.) Woods.

finitima Woods.

megagros (Vell.) Woods.

solanifolia (Müll. Arg.) Woods.

trifida (Poepp.) Woods.

Rauwolfia

paraensis Ducke

pentaphylla Ducke

polyphylla Benth.

sprucei Müll. Arg.

ternifolia H.B.K. (R. ligrestrina R. et. S.)

Rhabdadenia

macrostoma (Benth.) Müll. Arg.

Rhipidia

amazonica Mgf. 
Salpinctes

kalmiifolius Woods.

Secondatia

densiflora DC.

Stemmadenia

obovata (Hook. \& Arm.) K. Schum.

Stenosolen

heterophyllus (Vahl.) Mgf.

Tabernaemontana

amygdalifolia Mgf. (Colombia)

Temnadenia

ornata (Hoehne) Woods.

\section{SUMMARY}

A list of Apocynaceae species in the herbarium of the Instituto Nacional de Pesquisas da Amazônia is presented together with the corresponding results of a test indicating the presence or lack of alkaloids, the chemical alkaloidical characteristics of this family are also discussed, with emphasis on genero from the Americas.

\section{REFERENCIAS BIBLIOGRÁFICAS}

ALLEN, J.R.F. \& HOLMSTEDT, Bo R.

1980 - The simple $\beta$-carboline alkaloids Phytochemistry, 19 (8): 1573-1582.

CROQUELOIS, G.; KUNESCH, N.; DEBRAY, M.; POISSON, J.

1972 - Alstonia boonei alkaloids. Plant. Med. Phytother, 6 (2): 122-127.

DALLA TORRE, C.G. de \& HARMS, H.

1963 - Apocynaceae. In: genera siphonogamarum ad systema Englerianum conscripta. Wiesbaden Verlag, Berlin (reimpressão): 404-410.

HABERMEHL, G.G.

1973 - Steroid alkaloids. In: MTP International Review of Science. Alkaloids. Organic Chemistry, Series one. Ed. Wiernes, K. HESSE, $M$. Vol. 9: 250-257. Butterworths, London.

1964/1968 - Indolalkaloids in Tabellen. Idem Ergänzungswerk Springer - Verlag, Berlin.
MARKGRAF, F.

1976 - Apocynacées, $1699^{\circ}$ famille. Flore de Madagascar et des Comores, 1-318. Muséum National d'Histoire Naturalle, Paris.

MELCHIOR, H.

1964 - Apocynaceae. In: A. Engler, Syllabus der Pflanzenfamilien. G. Bonntraeger, Berlin. Ed. 12. 2: $411-414$.

PICHON, M.

1948 - Classification des Apocynacées, IX. Rau volfiées, Alstoniées et Tabernaemontanoidées. Mem. Mus. Nat. Hist. Nat. Paris, n-ser. 27 (6): 153-252.

RAFFAUF, R.F.

1970 - A Handbook of Alkaloids and Alkaloid Containing Plants. Wiley-Interscience, New York.

ROCHA, A.I. da

1977 - Alcalóides indoloterpênicos como marcadores quimiossistemáticos. Tese de Doutoramento, Universidade de São Paulo.

ROCHA, A.I. da; SILVA, M.L. da; A.P. \& CAVA, M.P.

1968 - A presença de alcalóides em espécies botânicas da Amazônia. Boletim do INPA, Série Química (12)

ROCHA, A.I. da; REIS LUZ, A.I. \& RODRIGUES, W.A.

1981 - A presença de alcalóides em espécies botânicas da Amazônia. III. Annonaceae. Acta Amazonica, 11 (3)

(Aceito para publicação em $30 / 04 / 82$ ) 